\title{
Experiences of Patients Undergoing Bowel Preparation and Colonoscopy: A Qualitative Longitudinal Study
}

This article was published in the following Dove Press journal: Journal of Multidisciplinary Healthcare

\author{
Sara Shamim ${ }^{\prime}$ \\ Yvette Lena Margareta \\ Andresen' \\ Henriette Vind Thaysen $\left(\mathbb{D}^{2}\right.$ \\ Ida Hovdenak Jakobsen ${ }^{2}$ \\ Jannie Nielsen ${ }^{\prime}$ \\ Anne Kjaergaard Danielsen (iD ' \\ Hanne Konradsen (D) \\ 'Department of Gastroenterology, \\ Herlev and Gentofte University Hospital, \\ Herlev, Denmark; ${ }^{2}$ Department of \\ Gastrosurgery, Aarhus University \\ Hospital, Aarhus, Denmark
}

Introduction: Colonoscopy remains the reference standard for diagnosing and monitoring colorectal cancer and for diagnosis and surveillance of inflammatory bowel disease. However, there is a limited knowledge of the patients' needs when undergoing colonoscopy and the challenges within in order to reduce the number of cancelled colonoscopies. The purpose of the study was to explore the experiences of undergoing bowel preparation and colonoscopy.

Methods: The study was designed as a qualitative longitudinal interview study with an inductive research approach. Patients were considered for inclusion consecutively and selected based on the following criteria of variation: way of referral for colonoscopy (outpatient or screening), age and gender. The interviews were analyzed using qualitative content analysis, and results reported according to COREQ guidelines.

Results: Based on the findings, three categories emerged: To weigh up participation, A greater challenge than expected, and Not so challenging after all. Throughout these categories, the experience of uncertainty was reported.

Discussion: The process of undergoing bowel preparation and colonoscopy was influenced by uncertainty due to insufficient information. There is a need to strengthen the patientcentered care by adjusting the information to patient's needs to better support them in decision-making for participation, to better prepare them for the bowel preparation and to better prepare them for the procedure. In addition, it is vital that patients are provided with results of the colonoscopy that correspond to the timeframe specified in written information. Keywords: colonoscopy, patient experience, qualitative

\section{Background}

Colonoscopy remains the reference standard method for diagnosing and monitoring colorectal cancer, ${ }^{1}$ and is frequently used for both diagnosis and surveillance of inflammatory bowel disease. ${ }^{2}$ Colonoscopy provides a visual examination of the rectum and colon to determine the presence of abnormalities and gives the opportunity for biopsy or removal of lesions. ${ }^{3}$ Anxiety and concerns, however, have been associated with the colonoscopy procedure. Being referred to the procedure can make patients feel anxious about the examination, about potential pain and embarrassment during the procedure and about the result of the examination. ${ }^{4-7}$

Whether it is outpatients or screening patients, they must undergo a scheduled bowel preparation up to 5 days prior to the colonoscopy, consisting of dietary restrictions such as not eating fiber-rich foods and dairy products and ingesting
Correspondence: Hanne Konradsen Herlev and Gentofte University Hospital, Borgmester lb Juulsvej, Herlev, 2730, Denmark

Email Hanne.konradsen@regionh.dk
Journal of Multidisciplinary Healthcare 2021:14 349-358

DovePress $f$ in $D$ 
large amounts of fluid and purgative solutions for colon cleansing. ${ }^{8}$ A review from 2012 showed that the laxative bowel preparation before colonoscopy was experienced as burdensome and that patients had many concerns related to pre-test fasting, restricted diet, and frequent visits to the bathroom. ${ }^{6}$

Due to an inadequate prepared colon, up to $20-30 \%$ of colonoscopies have been reported to become canceled. ${ }^{1}$ This can be burdensome for patients because they must repeat the bowel cleansing and the colonoscopy at another time and can contribute to an increase in societal costs.

Nurses have an important role in caring for patients referred to a colonoscopy; they give instructions for the preparatory phase, they assist during the procedure and make sure the patient feels safe, respected and taken care of, and they are in contact with patients in the postprocedural phase in case of patients feeling discomfort. Having a person-centered approach seems to have an important role for patients when evaluating quality of care, and this includes knowledge and communicative competences and timeliness of staff. ${ }^{9}$ The perspective of patients undergoing colonoscopy, from preparing for the procedure and until being back in everyday life again, is therefore important in order to know how to develop quality of care and reduce numbers of patients having their procedure cancelled.

The aim of this study was to explore experiences of undergoing bowel preparation and colonoscopy among outpatients.

\section{Methods}

\section{Design}

The study was designed as a qualitative longitudinal interview study with an inductive research approach, and data were analyzed using content analysis. ${ }^{10,11}$

\section{Setting}

The study was conducted from October 2019 to February 2020 at two departments of gastroenterology in Denmark. At both departments, a purgative solution for colon cleansing was used.

\section{Data Collection}

As the study sought to gain detailed descriptions of the experiences and thereby identify common patterns when undergoing colonoscopy, patients were considered for inclusion consecutively, and selected based on the following criteria of variation: way of referral for colonoscopy (outpatient or screening), age and gender. Inclusion criteria were as follows: patients index undergoing colonoscopy, able to undergo bowel preparation at home, +18 years and able to speak and understand Danish.

In Herlev and Gentofte University Hospital, patients were included by assessing a record of scheduled colonoscopies by a clinical nurse specialist from the department of gastroenterology. In Aarhus University Hospital (anonymous), patients were included by assessing a record of scheduled colonoscopies by two clinical nurse specialists. Patients' medical charts were assessed after the patients gave their informed consent, which included publication of anonymized responses.

Patients who met the inclusion criteria were invited to two interviews. The first interview was conducted face to face and took place at the hospital an hour before the colonoscopy when the patients had not yet been given sedative medicine. Approximately 1-2 weeks after the colonoscopy, the second interview was conducted by telephone, at a time convenient for the patient.

A semi-structured interview guide was used to facilitate a variety of ways of understanding patients' views and to produce richer data. The interview guide (Supplementary Material) was developed to capture the patients' experiences and was pilot tested with patients before their colonoscopy. The interview guide was refined during the pilot interviews, by changing few words to improve understanding of medical terms among patients.

In the first interview, the interview guide focused on the experience of bowel preparation. The second interview included questions concerning the experience of colonoscopy. The interview guide contained few and open-ended questions. Follow-up questions such as "what do you mean by?" or "can you tell me more?" were used as ways to elicit patients' experiences.

To ensure consensus in the interview procedure at both departments the interview guide, the introduction and invitation to the interview was discussed before conducting the interviews.

First author (SS) conducted 20 interviews at the department of gastroenterology in Herlev and Gentofte University Hospital. Two clinical nurse specialists (HVT and IHJ) conducted a total of five interviews in the endoscopic unit at the department of gastrosurgery in Aarhus University Hospital. At both departments, interviews were conducted in a separate room to ensure privacy.

The follow-up interviews consisted of 21 telephone interviews. Four of the telephone interviews were not conducted due to the following reasons: two patients 
were not possible to reach; one patient's colonoscopy was interrupted early in the process and one patient had passed away.

In Herlev and Gentofte University Hospital face to face interviews and follow-up interviews, interviews were digitally recorded by the dictation program MIRSK which is a program used in the Capital Region of Denmark that securely stores the audio files. In Aarhus University Hospital interviews were recorded by VIC-media player with similar functions.

The duration of the face-to-face interviews was between 12 and $40 \mathrm{~min}$ and the duration of the telephone interviews was between 04 and $19 \mathrm{~min}$. The patients' age ranged from 23 to 80 years.

Table 1 shows characteristics of the patients.

\section{Analysis}

Data were analyzed using inductive content analysis. ${ }^{10}$ The analysis consisted of several steps. To obtain an overall understanding of content related to the aim of the study and to achieve immersion, audio recordings of face-to-face interviews and follow-up interviews were listened several times. Meaning units were coded while listening to the interviews and codes were then grouped according to the same central meaning and then abstracted into categories and subcategories. We used the analytic approach to follow data both across patients and longitudinal following the single patient.

In this study, SS and HK listened to all interviews. The interviews were initially analyzed by SS and $\mathrm{HK}$, and

Table I Characteristics of the Patients

\begin{tabular}{|l|l|}
\hline & N $=\mathbf{2 5}$ \\
\hline $\begin{array}{l}\text { Gender } \\
\text { Female }\end{array}$ & 12 \\
Male & 13 \\
\hline Age distribution (years) & \\
$20-29$ & 2 \\
$30-39$ & 3 \\
$40-49$ & 1 \\
$50-59$ & 6 \\
$60-69$ & 6 \\
$70-79$ & 6 \\
$80-90$ & 1 \\
\hline Referral & \\
Outpatient (inflammatory bowel disease and colorectal & 15 \\
cancer) & 10 \\
Screening & \\
\hline
\end{tabular}

extracted codes were then discussed with the research team for validation. Categories and subcategories were then developed and discussed back and forth, until the research team reached a common understanding. ${ }^{12}$ Figure 1 provides an example of the analytic procedure.

To achieve credibility both codes and categories were discussed in the research team to discover less obvious points and to achieve agreement. Moreover, to establish credibility exemplary quotations from the interviews were included. ${ }^{10}$ To facilitate transferability, the study gave a clear description of the context, recruitment, characteristics of participants, data collection and process of analysis.

The study was reported according to the Consolidated Criteria for Reporting Qualitative Research (COREQ) checklist for interviews ${ }^{13}$ (Supplementary file 1).

\section{Ethics Approval and Informed Consent}

The study was approved by the Danish Ethics committee, Capital region (H-19049983). The Danish Data Protection Agency was consulted, and formal consent was given (P-2019-333). Prior to colonoscopy, the patients were given oral and written information about the study. Both oral and written consent was obtained from the patients, all participants provided informed consent to have their anonymized responses published, in accordance with the Declaration of Helsinki. All data material was treated and stored confidentially.

\section{Results}

Based on the findings, three categories with related subcategories emerged: To weigh up participation, A greater challenge than expected, and Not so challenging after all. As this study used a longitudinal design, findings were presented to understand how patients' experiences evolved through the process.

\section{To Weigh Up Participation}

Considering participation in the colonoscopy was affected by an uncertainty of not knowing the conclusion of the procedure and about the procedure itself. However, the professionalism of the health-care professionals (HP) was trusted.

\section{Uncertainty}

Receiving the invitation to colonoscopy and the patient guidelines for bowel cleansing made patients reflect. If the purpose of the colonoscopy was to affirm a cancer diagnosis, the statistics and numbers about cancer presented in the invitation was thought-provoking which made them 


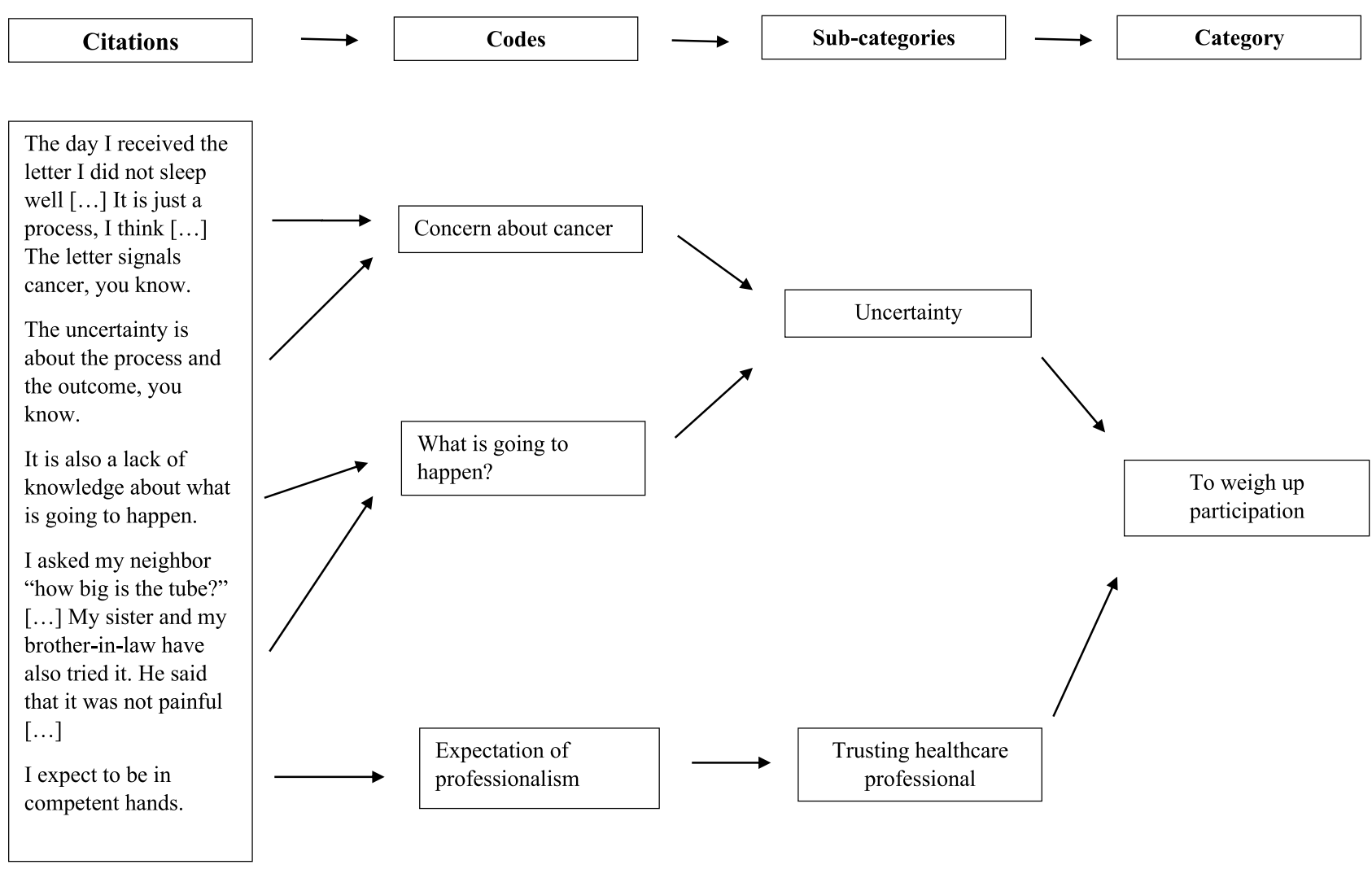

Figure I Illustration of citations and codes underpinning sub-categories and a category.

think about pros and cons for participating. But statistics could be reassuring because it affirmed that undergoing a colonoscopy not necessarily resulted in a cancer diagnosis. This made some patients assume to be amongst those who did not have a cancer diagnosis. Also, it drew their thoughts on the uncertainty of the results and the risk of having a cancer diagnosis, which made them uncomfortable. The speculations led them to think about their own or family members' experiences with cancer.

One patient used words like:

It was receiving the admission. It was stupid. My wife just died of cancer. A year ago, right. So, uhm, we are almost frequent visitors here [the hospital], right. (Patient 1)

Patients also experienced an uncertainty concerning the colonoscopy in general. As it was their first colonoscopy, they did not know enough about the procedure. They questioned the duration of the procedure, how they would lie down during the procedure, whether they would experience pain and the possibility to follow the procedure on screen. Moreover, patients expressed concerns about sedative medicine by saying:
It [the information leaflet, ed] stated that you would be given a sedative. So, I do not know whether it is a pill or a sting you get in your arm, which makes you sleep a little bit. (Patient 2)

To get answers to these questions they sought information themselves. They talked to relatives and their closest friends who had experienced a colonoscopy or looked for answers on the internet such as watching a video. This gave them an idea of what the bowel preparation and colonoscopy was about, which was both calming and worrying. The following quotation illustrated this:

The experience she had of it [the bowel preparation, ed] is completely different from what I have experienced. (Patient 1)

\section{Trusting Healthcare Professionals}

Patients had a clear expectation that the colonoscopy was performed by caring HP. They expected the HP to be kind, including, not rushing to complete the examination and to be competent. They were convinced that the HP would do their best as well:

I rely a lot on our health care. [...] they know what they are doing (Patient 2) 
The professionalism of the HP was of importance. However, there was a pervasive feeling of uncertainty about the result. Due to this, patients were both interested in and expected to be informed about the result at the end of the procedure. By knowing the result, they did not have to live with the uncertainty more than necessary. This was described by a patient like:

It is an uncertainty about it all [...] Well, I know I can close one door after this. (Patient 5)

Although participating in colonoscopy evoke the feeling of uncertainty patients expressed gratitude for being offered the colonoscopy. They were glad that there was a way to find out if something was wrong. They understood the importance of undergoing a colonoscopy and felt it to be in their own interest, but they also expressed an obligation to participate. A patient expressed this by saying:

After all, there is no way out. You have to. (Patient 4)

The risk in not undergoing a colonoscopy was too great to avoid for the patients. The bowel cleansing and colonoscopy was for the sake of a greater good. If that was what it took to be sure they would do it and they wanted it to be over with.

\section{A Greater Challenge Than Expected}

Preparing for the colonoscopy affected daily life and was more comprehensive than expected. Following the instructions was challenging and provided an uncertainty about following them correctly.

\section{Preparation in Daily Life}

The bowel preparation was a challenge. The dietary restrictions were overwhelming and experienced as restrictive because they did not allow patients to eat what they generally preferred eating. Consequently, patients constantly felt a lack of satiety. A patient expressed her frustration with the dietary change like:

I think that was terrible. I spent the first whole week wondering how I should get any food at all. (Patient 6)

The dietary change was also challenging because they were unaware of the reason for the dietary restrictions and how it could affect the colonoscopy. Additionally, it was tiresome for them to follow a diet plan and they spent a lot of energy adapting to it. The diet changes required planning in daily life. Preparing and eating the special diet also had to be planned in relation to meals for the rest of the family. Patients had to pay special attention to meals at work, so they for example did not risk eating grain. To make sure that they did not eat something that was not stated in the diet plan they brought packed lunches to work.

During the bowel, preparation patients were challenged by the bowel cleansing procedure as well. The cleansing liquid was hard to drink. They described it as having a sweet and synthetic taste that was nauseating and a consistency making it hard to drink. Also, they had to drink large amounts of it in a short time, making it more difficult to plan their meals and affecting their sleep. A patient highlighted it by saying:

I could not drink it all yesterday. Maybe I threw out a deciliter the first time. Then I had to get up in the middle of the night at 04 to start the next portion. So, I almost did not sleep because I was constantly on the toilet. (Patient 7)

The bowel preparation disrupted the daily life. This was also related to the frequent toilet visits. A patient described her experience with the toilet visits as:

I had to be careful all the time. So, you could feel that it was coming now and then you had to run to the toilet immediately [...] I had no control of when it was coming. (Patient 3)

Toilet visits required a lot of coordination as, patients were unsure of being able to hold stool back. Therefore, whether at home or at work they made sure they were close to a toilet. In addition, visiting the toilet frequently was painful and they ended up feeling skinless around the rectum.

\section{To Follow the Instructions}

The patients had confidence in the instructions and followed them closely during the bowel cleansing process. Although there was a lot of information in the instructions to consider, It was difficult to know when to do what. This made them reflect on how challenging it might be for elders or less resourceful people to perform the bowel cleansing properly.

They expressed a great desire for a clearer overview and created their own ways to manage the instructions. To better follow the guideline, simplified strategies were used such as reading guidelines several times and letting relatives take responsibility of the bowel cleansing. In the following citation, a patient described his wife having a big part in the bowel cleansing process: 
I have a wife who has taken care of the practical part. [...] It is actually a huge help. I do not envy those who are all alone with something like this. (Patient 8)

Simultaneously, patients felt that the instructions were deficient. They were very careful about not doing anything wrong and expected their body to respond to the bowel cleansing exactly as described in the instructions. When the body reacted differently than described in the instructions, patients doubted whether they had done the bowel cleansing properly. They expressed that they could be better prepared for the bowel cleansing if the instructions provided more details on side effects and what they could expect during the bowel cleansing. Also, they needed more ideas for meals. A patient described her experience as follows:

I tried to be creative with the things that were listed.

I would like if there were more things listed that you could eat, because I was in doubt about some things.

(Patient 9)

Different strategies were made to manage food preparation and patients felt that they had to invent creative ways to prepare a meal with the few ingredients that were stated in the guideline. In relation to this, they often doubted which vegetables and fruits to eat.

\section{Not so Challenging After All}

Undergoing the colonoscopy was a better experience than expected. The behavior of HP provided comfort and patients felt less uncomfortable undergoing a colonoscopy in the future.

The colonoscopy was not as unpleasant as expected and during the colonoscopy, they received a temporary evaluation on the examination, which was relieving. This experience led them to reassess their experience with the bowel cleansing and to reflect on the true meaning of the colonoscopy. In retrospect, they did not feel the bowel cleansing or colonoscopy to be that challenging and saw the cleansing as a bagatelle compared to what other people went through.

The HP also contributed to a better experience with the colonoscopy than expected. The patients considered them to be competent and they were good at explaining what happened during the procedure. Being exposed during the colonoscopy was transgressive for patients. It made them uncomfortable to think about where the tube was inserted. However, the HP covered them when possible and they felt that the HP's behavior in general made the colonoscopy less uncomfortable. The HP took care of their needs and were caring. The HP distracted the patients with small talk as well. It soothed them and shifted the focus of the colonoscopy. The use of humor by the HP also facilitated a diversion. This was described by a patient like:

[...] and then we laughed a little. So, I think there was a nice atmosphere [...] It loosened up the mood you were going in to. (Patient 10)

Overall HP made the patients feel safe. The feeling of safety could be disturbed during the colonoscopy though. One example was when another physician was called in to perform the colonoscopy, the patients questioned the experience and skills of the first physician. The following quotation illustrated this:

When the other physician came, he could just fix it. It makes me wonder that it required another physician. [...] It would have been fine if I had agreed that they could try it [the colonoscopy] for the first time, but I certainly did not. (Patient 7)

Another example was when they were not informed about another physician performing the colonoscopy. Furthermore, the other physician did not introduce himself. Consequently, the patients felt ignored.

Although the above situation left a bad impression on the patients, it did not affect their overall experience with the colonoscopy. Their experience made them feel better prepared for a colonoscopy in the future and they felt less uncomfortable by the thought of it.

The patients still felt that whether the experience of the colonoscopy was positive or negative, depended on the result. They expected to get the result shortly after, because this was stated in the instructions. However, their expectation of response time was not fulfilled. Patients waited several weeks for a response. It made them uncomfortable and they expressed frustration by the long wait.

\section{Discussion}

The aim of the study was to explore experiences of undergoing bowel preparation and colonoscopy, with the purpose of contributing to develop the quality of care and find ways of reducing cancelled colonoscopies.

The findings resulted in three categories highlighting that patients weighed up whether to participate in the colonoscopy or not, that preparations for the colonoscopy 
was a greater challenge than expected and that in retrospect, the colonoscopy was not so challenging after all. Throughout these categories, the experiences of uncertainty could be recognized. Theory of Uncertainty in illness by Mishel (1988) ${ }^{14}$ can serve as a way of describing some of our findings. According to Mishel (1988), uncertainty is the inability to attribute meaning to illness-related events and to predict outcomes. Mishel specifies that disease complexity, poor information provision and the unpredictability or ambiguity of events affect the ability to confer meaning and thereby increases uncertainty. We found that poor information provision contributed to the uncertainty experience as patients had difficulties understanding and predicting the requirements for the bowel preparation and colonoscopy when undergoing the procedure for the first time. Additionally, unpredictability occurred when rapid result delivery was not experienced after the colonoscopy. The patients who were examined for cancer might have experienced the colonoscopy as an opportunity to obtain reassurance but also as evoking fear of cancer proximity. This appeared by patients' uncertainty about participating in the colonoscopy and it made them reflect and weigh up the benefits and disadvantages of it. Weighing the benefits and disadvantages of colorectal cancer screening (CRS) before deciding on participation has been shown in other studies ${ }^{15,16}$ Also, the use of the social network to discuss has shown to increase the willingness to participate in CRS. ${ }^{17}$ The importance of the social network was found in our study as well. However, sharing thoughts in the social network was both calming and worrying. Depending on the experiences of patients' social network, it either reduced patients' uncertainty about the procedure or increased it.

It seemed that patients weighing was affected by different factors, which was described in other contexts as well. ${ }^{18,19}$ Overall, results suggested that in-depth information about the colonoscopy and reasons for participating was needed.

Besides weighing benefits and harms of undergoing colonoscopy, patients felt obliged to participate. In screening programs, patients had felt compelled to do as told when receiving a screening invitation and to participate for the sake of the family. ${ }^{20-22}$ An interesting finding from the current study was though that patients also expressed obligation to participate because they felt there was no other way for illness detection. Thus, participation in colonoscopy may not solely be depending on patients' own opinions although it is for the benefit of the patient.
These circumstances, however, emphasized the importance of giving patients the best conditions for decision-making.

Colonoscopy was regarded as necessary but one that interfered with patient's daily life. The daily life was affected when managing the bowel preparation making it more challenging than expected. Previous research has also found that including bowel preparation in everyday life is difficult for patients undergoing colonoscopies. ${ }^{23,24}$ These challenges have even shown to exist in patients who have experienced multiple colonoscopies. ${ }^{25}$ Although planning in everyday life due to participation has also shown to be challenging in other screening settings like breast cancer screening, ${ }^{26,27}$ preparation for colonoscopy has shown to interfere with everyday activities for a longer period.

In the present study, following instructions during bowel preparation process was difficult. The bowel preparation has been described as a barrier to screening. 1,23,24 However, our findings revealed new aspects by showing how large amount of information in the instructions and undergoing a scheduled bowel preparation with dietary restrictions challenged the preparation and patients' concern about not performing the preparation properly due to instructions. This showed a need of exploring more effective ways to convey information.

Looking back, patients in the current study experienced that undergoing colonoscopy was not as challenging as expected. This is in line with previous findings showing that colonoscopy expectations were more negative compared to post-colonoscopy appraisals in terms of pain, unpleasantness and embarrassment. ${ }^{6,28}$

Medical skills and a caring behavior of the HP have shown to be important when undergoing screening. ${ }^{21,25,29}$ These competences were also valued and experienced by our patients, which contributed to a less challenging colonoscopy experience. The competences were particularly needed when exposing the lower part of the body. Not being in control of bodily functions and being unable to conceal the body can result in increased vulnerability and embarrassment during this procedure, which makes a caring behavior crucial. ${ }^{22,25}$ Furthermore, embarrassment among male patients can be caused by a negative sexual connotation to the procedure. ${ }^{30}$

Distracting patients by using humor during colonoscopy also made it less challenging for them. Use of humor by HP can help patients cope with the unpleasant and discomforting situation the colonoscopy might cause. Though humor is both personal and multifaceted that can 
cause discomfort if not used appropriately. ${ }^{31}$ Similar coping mechanisms have been used by patients to minimize feelings of shame and physical discomfort in other healthcare contexts. ${ }^{19,25}$

Our findings indicated, as resonated in previous research, ${ }^{25,27,32}$ that delay in receiving test result was unpleasant. The unpleasantness can both be an expression of this delay being unexpected and an expression of their uncertainty about what to expect from the test results. This implied a need for clear and concise information including reasons for unexpected events such as longer waiting times.

\section{Strengths and Limitations}

The findings of this study provide insights from the patient's perspective. These can be leveraged to improve the experience of undergoing bowel preparation and colonoscopy and be transferable into similar contexts. However, the findings should be interpreted within its strength and limitations.

In this study, the interviews were not transcribed. Though transcribing interviews is a way to reproduce spoken words and facilitate data analysis, the process of transcription is open to transcriber errors and time consuming. ${ }^{33}$ However, analyzing data directly from recordings made it possible to capture nuances on words and reduced misinterpretation of content. ${ }^{34}$ Therefore, this analysis technique provided rigorous findings in the current study. Furthermore, all participating researchers listened to the interviews and as such the results were validated in the author group.

Conducting the study at two departments of gastroenterology increased the validity of findings. According to the clinical nurse specialists from the research team, the findings were relatable to clinical practice. This also validated the findings, as a form of external validity. ${ }^{35}$ However, this study excluded patients who were not fluent in Danish. A language barrier could make undergoing colonoscopy more challenging for these patients. Thus, how our findings would hold among ethnically diverse patients is unknown.

The fact that follow-up interviews were conducted 2 weeks after the colonoscopy and because some patient's colonoscopy procedure was carried out under mild sedation, oversight and recall biases of relevant experiences cannot be ruled out. Moreover, patient's nervousness prior to the colonoscopy procedure may have influenced their responses during the face-to-face interviews.
However, the experiences described were rich in content and participants were able to answer the research question.

\section{Conclusion}

Undergoing bowel preparation and colonoscopy was a multifaceted process for the patients. During this process, their experiences were found to be influenced by uncertainty which occurred when deciding to participate, during the bowel preparation and not knowing the result in a timely manner. Though patients tried to manage the uncertainty by using different strategies, it left them feeling an unpredictability of the process due to insufficient information. However, patients experienced medical skills and a caring behavior of HP during colonoscopy which was crucial.

These findings underline the importance of focusing on patient's perspective in the clinical setting and in information provision including a better understanding of how uncertainty impacts on patients before, during and after completing a colonoscopy. Future studies are needed to explore how information provision can be adjusted to patients' needs and impact patient experienced outcomes and the participation in the procedure.

\section{Implication for Practice}

Our findings should create awareness of how uncertainty influences patients when undergoing bowel preparation and colonoscopy and that the importance of patient information provision cannot be overemphasized. New ways must be discovered to better inform and initiate patientcentered care in order to improve bowel preparation and reduce the numbers of cancelled colonoscopies. In particular, the received written information must be tailored to meet patients' needs to better support them when deciding to participate, to better prepare them for the bowel preparation including ideas for managing food preparation and to better prepare them for the procedure. This might reduce preprocedural uncertainty. A way to accomplish this can be by implementing more interactive audiovisual methods like a smartphone app, that have shown to improve bowel preparation and patient adherence compared to standard patient education. ${ }^{36}$

In addition, it is necessary that patients are provided the results of colonoscopy that correspond to the timeframe specified in the written information and that all patients are provided results whatever the results might be to minimize the uncertainty experienced after the colonoscopy. 


\section{Abbreviations}

HP, healthcare professionals; CRS, colorectal cancer screening.

\section{Data Sharing Statement}

Data sharing is not applicable to this article as sharing sound recordings is illegal in accordance with Danish law.

\section{Acknowledgments}

We would like to thank the patients for their time and contributions to the study.

\section{Funding}

The study did not receive any specific grant from any funding agencies in the public, commercial, or not-forprofit sectors.

\section{Disclosure}

The authors report no conflicts of interest related to this work.

\section{References}

1. sLiu Z, Zhang MM, Li YY, Li LX, Li YQ. Enhanced education for bowel preparation before colonoscopy: a state-of-the-art review. J Dig Dis. 2017;18(2):84-91. doi:10.1111/1751-2980.12446

2. Restellini S, Kherad O, Bessissow T, et al. Systematic review and meta-analysis of colon cleansing preparations in patients with inflammatory bowel disease. World $J$ Gastroenterol. 2017;23 (32):5994-6002. doi:10.3748/wjg.v23.i32.5994

3. Ylinen ER, Vehviläinen-Julkunen K, Pietilä AM. Effects of patients' anxiety, previous pain experience and non-drug interventions on the pain experience during colonoscopy. $J$ Clin Nurs. 2009;18 (13):1937-1944. doi:10.1111/j.1365-2702.2008.02704.x

4. Bhise V, Modi V, Kalavar A, et al. Patient-reported attributions for missed colonoscopy appointments in two large healthcare systems. Dig Dis Sci. 2016;61(7):1853-1861. doi:10.1007/s10620-016-4096-3

5. Yang C, Sriranjan V, Abou-Setta AM, Poluha W, Walker JR, Singh H. Anxiety associated with colonoscopy and flexible sigmoidoscopy: a systematic review. Am $J$ Gastroenterol. 2018;113:1810-1818. doi:10.1038/s41395-018-0398-8

6. McLachlan SA, Clements A, Austoker J. Patients' experiences and reported barriers to colonoscopy in the screening context-A systematic review of the literature. Patient Educ Couns. 2012;86(2):137-146. doi:10.1016/j.pec.2011.04.010

7. Rollbusch N, Mikocka-Walus AA, Andrews JM. The experience of anxiety in colonoscopy outpatients: a mixed-method study. Gastroenterol Nurs. 2014;37(2):166-175. doi:10.1097/SGA.0000000000000037

8. Liang Z, Richards R. Virtual colonoscopy vs optical colonoscopy. Expert Opin Med Diagn. 2010;4(2):159-169. doi:10.1517/ 17530051003658736

9. Edvardsson D, Watt E, Pearce F. Patient experiences of caring and person-centredness are associated with perceived nursing care quality. $J$ Adv Nurs. 2017;73(1):217-227. doi:10.1111/jan.13105

10. Graneheim UH, Lundman B. Qualitative content analysis in nursing research: concepts, procedures and measures to achieve trustworthiness. Nurse Educ Today. 2004;24(2):105-112. doi:10.1016/j.nedt.2003.10.001
11. Saldaña J. Longitudinal Qualitative Research. Analyzing Change Through Time. Rowman \& Littlefield Publishers, Inc.; 2003.

12. Graneheim UH, Lindgren BM, Lundman B. Methodological challenges in qualitative content analysis: a discussion paper. Nurse Educ Today. 2017;56:29-34. doi:10.1016/j.nedt.2017.06.002

13. Tong A, Sainsbury P, Craig J. Consolidated criteria for reporting qualitative research (COREQ): a 32-item checklist for interviews and focus groups. Int J Qual Health Care. 2007;19(6):349-357. doi:10.1093/intqhe/mzm042

14. Mishel MH. Uncertainty in illness. Orthop Nurs. 1988;22 (3):206-214. doi:10.1097/00006416-200305000-00009

15. Flocke SA, Stange KC, Cooper GS, et al. Patient-rated importance and receipt of information for colorectal cancer screening. Cancer Epidemiol Biomarkers Prev. 2011;20(10):2168-2173. doi:10.1158/ 1055-9965.EPI-11-0281

16. Van Dam L, Korfage IJ, Kuipers EJ, et al. What influences the decision to participate in colorectal cancer screening with faecal occult blood testing and sigmoidoscopy? Eur J Cancer. 2013;49 (10):2321-2330. doi:10.1016/j.ejca.2013.03.007

17. Fritzell K, Stake Nilsson K, Jervaeus A, Hultcrantz R, Wengström Y. The importance of people's values and preferences for colorectal cancer screening participation. Eur J Public Health. 2017;27 (6):1079-1084. doi:10.1093/eurpub/ckw266

18. Schonberg MA, McCarthy EP, York M, Davis RB, Marcantonio ER. Factors influencing elderly women's mammography screening decisions: implications for counseling. BMC Geriatr. 2007;7:1-8. doi:10.1186/1471-2318-7-26

19. Whelehan P, Evans A, Ozakinci G. Client and practitioner perspectives on the screening mammography experience. Eur J Cancer Care (Engl). 2017;26(3):1-12. doi:10.1111/ecc.12580

20. Griffiths F, Bendelow G, Green E, Palmer J. Screening for breast cancer: medicalization, visualization and the embodied experience. Health (Irvine Calif). 2010;14(6):653-668. doi:10.1177/ 1363459310361599

21. Kirkegaard P, Edwards A, Andersen B. A stitch in time saves nine: perceptions about colorectal cancer screening after a non-cancer colonoscopy result. Qualitative study. Patient Educ Couns. 2019;102(7):1373-1379. doi:10.1016/j.pec.2019.02.025

22. Toft EL, Kaae SE, Malmqvist J, Brodersen J. Psychosocial consequences of receiving false-positive colorectal cancer screening results: a qualitative study. Scand J Prim Health Care. 2019;37 (2):145-154. doi:10.1080/02813432.2019.1608040

23. Chopra D, Hookey LC. Comorbid illness, bowel preparation, and logistical constraints are key reasons for outpatient colonoscopy nonattendance. Can J Gastroenterol Hepatol. 2016;2016:11-15. doi:10.1155/2016/2179354

24. Sultan S, Partin MR, Shah P, et al. Barriers and facilitators associated with colonoscopy completion in individuals with multiple chronic conditions: a qualitative study. Patient Prefer Adherence. 2017;11:985-994. doi:10.2147/PPA.S127862

25. Ryhlander J, Ringstrom G, Simrén M, Stotzer P-O, Jakobsson S. Undergoing repeated colonoscopies - experiences from patients with inflammatory bowel disease. Scand J Gastroenterol. 2019;54 (12):1467-1472. doi:10.1080/00365521.2019.1698649

26. Mathioudakis AG, Salakari M, Pylkkanen L, et al. Systematic review on women's values and preferences concerning breast cancer screening and diagnostic services. Psychooncology. 2019;28(5):939-947. doi:10.1002/pon.5041

27. Norfjord Van Zyl M, Akhavan S, Tillgren P, Asp M. Experiences and perceptions about undergoing mammographic screening: a qualitative study involving women from a county in Sweden. Int J Qual Stud Health Well-Being. 2018;13(1):1521256. doi:10.1080/17482631.20 18.1521256

28. Condon A, Graff L, Elliot L, Ilnyckyj A. Acceptance of colonoscopy requires more than test tolerance. Can J Gastroenterol. 2008;22 (1):41-47. doi:10.1155/2008/107467 
29. Mathers SA, McKenzie GA, Robertson EM. "It was daunting": experience of women with a diagnosis of breast cancer attending for breast imaging. Radiography. 2013;19(2):156-163. doi:10.1016/ j.radi.2012.11.004

30. Bynum SA, Davis JL, Green BL, Katz RV. Unwillingness to participate in colorectal cancer screening: examining fears, attitudes, and medical mistrust in an ethnically diverse sample of adults 50 years and older. Am J Health Promot. 2012;26(5):295-300. doi:10.4278/ ajhp.110113-QUAN-20

31. Sousa LMM, Marques-Vieira CMA, Antunes AV, Frade M de FG, Severino SPS, Valentim OS. Humor intervention in the nurse-patient interaction. Rev Bras Enferm. 2019;72(4):1078-1085. doi:10.1590/ 0034-7167-2018-0609

32. Bailey DE, Wallace M, Mishel MH. Watching, waiting and uncertainty in prostate cancer. J Clin Nurs. 2007;16(4):734-741. doi:10.1111/j.1365-2702.2005.01545.x
33. Halcomb EJ, Davidson PM. Is verbatim transcription of interview data always necessary? Appl Nurs Res. 2006;19(1):38-42. doi:10.1016/j.apnr.2005.06.001

34. Greenwood M, Kendrick T, Davies H, Gill FJ. Hearing voices: comparing two methods for analysis of focus group data. Appl Nurs Res. 2017;35:90-93. doi:10.1016/j.apnr.2017.02.024

35. Konradsen H, Kirkevold M, Olson K. Recognizability: a strategy for assessing external validity and for facilitating knowledge transfer in qualitative research. Adv Nurs Sci. 2013;36(2):66-76. doi:10.1097/ ANS.0b013e318290209d

36. Desai M, Nutalapati V, Bansal A, et al. Use of smartphone applications to improve quality of bowel preparation for colonoscopy: a systematic review and meta-analysis. Endosc Int Open. 2019;07 (02):E216-E224. doi:10.1055/a-0796-6423
Journal of Multidisciplinary Healthcare

\section{Publish your work in this journal}

The Journal of Multidisciplinary Healthcare is an international, peerreviewed open-access journal that aims to represent and publish research in healthcare areas delivered by practitioners of different disciplines. This includes studies and reviews conducted by multidisciplinary teams as well as research which evaluates the results or conduct of such teams or healthcare processes in general. The journal
Dovepress

covers a very wide range of areas and welcomes submissions from practitioners at all levels, from all over the world. The manuscript management system is completely online and includes a very quick and fair peer-review system. Visit http://www.dovepress.com/testimonials. php to read real quotes from published authors. 